\title{
DIRECTION FINDING AND POLARIZATION MEASUREMENTS OF SKR
}

\author{
A. Lecacheux*
}

\begin{abstract}
The Saturnian Kilometric Radio emission (SKR), discovered and briefly observed by Voyager spacecraft in 1980-81, is now studied in depth by Cassini, which is still in orbit around Saturn, since mid 2004.

Aboard Cassini, the main radio astronomy system (the HFR part of the RPWS instrument) is based on digital, real time, spectral correlation of several pass band filters, analyzing multiple wire antennas. This system allows, in principle, the full second order statistics of the analyzed signal to be retrieved, thus providing, compared to a simple antenna system, some extra information on the received radio waves: mainly about the spatial brightness distribution (leading to direction finding (DF) for a point source model) and the intrinsic polarisation of the measured radio source.

While there is no doubt that SKR, like terrestrial and Jovian radio emissions, is powered via a cyclotron maser (CMI) originating from accelerated auroral particles, the exact scenario remains, at the moment, far from being understood: the complex modulation of the SKR at the planetary spin rate is perplexing; the fact that the apparent SKR polarization changes with observer's latitude and contains a substantial amount of linear polarisation when observed from mid to high latitudes, is quite unexpected and likely the indication of a complex source structure and/or unusual propagation regime in Saturn's high latitude regions.
\end{abstract}

\section{Introduction}

Voyager spacecraft observations have established that the Saturnian Kilometric radiation (SKR) is a non thermal, coherent radio emission generated by electron beams which are accelerated above auroral zones of Saturn by a cyclotron maser instability [Kaiser et al., 1984]. The emission was observed as being radiated in the X-mode near local electron gyro frequency, within a frequency range from a few to about $1200 \mathrm{kHz}$. The radiation is highly directive, is emitted at large angle to the local magnetic field, and is purely circularly polarized (in right-handed and left-handed senses from Northern and

* LESIA, Observatoire de Paris, UMR CNRS 8109, 92195 Meudon, France 
Southern hemisphere, respectively), as expected from outwards quasi-longitudinal (QL) wave propagation.

After Voyager, some questions remained open, regarding the relationship of SKR with Saturn auroras and the generation mechanism of radio emission. What are the exact locations of SKR radio source(s), in particular with respect to those of Saturn UV/IR auroras? Why does SKR exhibit the periodic modulation of a clock-like triggered source [Warwick et al., 1981]? What is the actual beaming and polarisation? How can the observed various time scales and spectral fine structures of SKR be explained?

New responses should be provided by Cassini, in orbit around Saturn since 2004, July $1^{\text {st }}$, owing to the very comprehensive parts of its payload, dedicated to study of Saturn's magnetosphere. In particular, the radio astronomy instrument possesses, for the first time in an outer planetary mission, the optimal capability permitted by the use of simple wire antennas erected on a spacecraft [Lecacheux et al., 1979].

Aboard Cassini, the main radio astronomy system - the HFR part of the RPWS instrument, [Gurnett et al., 2004] -, is based on digital, real time, spectral correlation of several pass band filters, analyzing a set of three wire antennas. This system allows, in principle, the full second order statistics of the analyzed signal to be retrieved, thus providing, with respect to a simple antenna system, some extra information on the received radio waves: mainly about the spatial brightness distribution (leading to direction finding (DF) for a point source model) and about the intrinsic polarisation of the measured radio source.

\section{How the RPWS Instrument Works}

The RPWS antenna system includes three 10-meter long monopoles (named 'u', 'v', 'w'), of which two can be combined to a dipole ('u-v'). With the third antenna ('w'), three possible pairs of antennas can be formed ('u,w', 'v,w', '(u-v),w'), which are eventually analyzed by digital receivers.

At each frequency step of a spectral sweep, the RPWS/HFR basic data sample is made of four quantities, proportional to the auto- and cross co-variances of the voltages from one pair of antennas. This working mode is known as the "2 antennas mode". It does not allow the full polarisation and direction, together, to be retrieved [Lecacheux et al., 1979; Cecconi et al., 2005].

This process can be immediately repeated with another antenna pair, in order to build an extended, eight quantities data sample. This working mode is known as the "3 antennas mode" or "DF mode". In the case of a steady emitting point source, this mode allows a full polarisation and direction analysis [Lecacheux, 1978]. In order to save telemetry bandwidth, the 3-antennas mode was not in operational use before 2006 (excepted during a few weeks in July and August 2004), then was operated about one third of the time since 2007.

Hereafter and for conciseness, the whole data processing operation dealing with retrieval of both arrival direction and polarisation of an incident wave will be designated by the term 'DF\&Pol'. 


\section{Some Already Published DF\&Pol Results on SKR}

Since the Cassini injection into Saturn's orbit in July 2004, several studies have already been published, which have started to apply some direction finding and polarisation retrieval methods to RPWS/HFR data.

Lamy et al. [2008] carried out a statistical study of all available radio data obtained from SOI until 2007. They confirmed most of the Voyager findings, in particular, a prevailing SKR visibility when Cassini was located on the morning side of Saturn's magnetosphere. They also measured a dominant X-mode emission, but found some additional O-mode component below $100 \mathrm{kHz}$.

Two case studies were later performed. Cecconi et al. [2009] studied twelve hours of data around the revolution 29 periapsis. By using data along this orbit, with a closest approach at $\sim 4 R_{S}$ and a relatively high inclination, they could estimate the beaming angle, the invariant latitude and the local time of some SKR sources. The estimated locations were found to match that of UV and IR auroras. On the other hand, Lamy et al. [2010,2011] took benefit of spacecraft crossing (2008 October 17) of some SKR southern sources, for measuring CMI emission angle and polarisation near the source. They found a mixture of $\mathrm{X}$ and $\mathrm{O}$-mode with different spectral coverages, a large beaming angle and a strong elliptical polarisation of the SKR near $10 \mathrm{kHz}$. So, the authors suggest that there are some significant differences in apparent SKR properties when observed from near or far distances.

The discovery by Fischer et al. [2009] that SKR polarization becomes strongly elliptical when observed from high latitudes, was even more puzzling. Indeed, from low latitude observations, it was firmly established [Kaiser et al., 1984] that SKR is totally and circularly polarized, and first observations by Cassini, from orbits in Saturn's equator, definitely confirmed this [Lamy et al., 2008]. Such a circular polarization state was also expected [Lecacheux, 1988], as a result of outwards QL wave propagation in Saturn's inner magnetosphere. In order to be observed elliptically polarized, the SKR initial polarization has to be retained in its original state (so elliptically polarized, implying quite low plasma density in the source) or further changed by strong mode coupling (for instance through a region of propagation transverse to the magnetic field), before to reach the observer. But it is unclear why that would happen as a function of observer's latitude above Saturn's equator.

All these first studies were very encouraging and confirm the pertinence of the RPWS/HFR instrumentation for studying Saturn's radio emissions. However, they also reveal some frustrating limitations of the utilized retrieval techniques. Most of them did not exploit the best available configurations of the instrument (3-antenna mode for instance); the actual accuracy of direction and polarisation parameter estimates were usually not provided. A typical example is given by Figure 5 of Fischer et al., [2009], in which a number of SKR source direction estimates were plotted, like an image over the sky, in projection onto a plane perpendicular to the line of sight, centred on Saturn, when Cassini was at $\sim 17 R_{S}$ from the planet. The accuracy quoted by the authors was $\sim 1 R_{S}$. All plotted directions, as raw outputs of the used estimation procedure, form a cloud of scattered points, more than $3 R_{S}$ in diameter, with some directions as far as $10 R_{S}$ from Saturn's 
centre. Looking at this figure, we certainly would know why apparent directions are so much scattered: is the dispersion due to retrieval method artefacts? Or due to some physical scattering occurring between Saturn and the observer? Is the radio source actually small or extended in size?

\section{Improving DF\&pol Retrieval Technique}

In order to improve the direction finding and polarisation retrieval technique, one ideally needs a complete mathematical model for describing the various stages of the internal HFR data processing. This involves the antenna transfer function, AGC operation, voltage digitization, frequency filtering (by digital convolution), and co-variances computation from voltage samples. In the following, this work will focus on the last step which mainly determines the final statistics of the data.

\subsection{Fundamentals on Electromagnetic Wave Polarization}

Modern notations for studying wave polarisation in radio astronomy have been introduced in theoretical analysis of the interferometer response of antenna arrays [Thompson et al., 2007; Hamaker et al., 1996]. In our case of several wire antennas erected on a spacecraft, we can use a similar formalism.

Given $\mathbf{E}(t)$, the electric field of an electromagnetic plane wave, its polarization can be interchangeably defined as the second order statistical moments of the electric field components (sample average), as the averaged electric field co-variances (time average) or as the wave coherence (e.g. Born et al., [1999]), leading to the following equivalent representations, by setting $\mathbf{E}=\left[E_{x} E_{y}\right]^{T}$, and using the usual notations of matrix calculus:

as coherent matrix:

$$
\mathbf{C}=\left\langle\mathbf{E E}^{\mathbf{H}}\right\rangle=\left(\begin{array}{cc}
\left\langle E_{x} \bar{E}_{x}\right\rangle & \left\langle E_{x} \bar{E}_{y}\right\rangle \\
\left\langle E_{y} \bar{E}_{x}\right\rangle & \left\langle E_{y} \bar{E}_{y}\right\rangle
\end{array}\right)
$$

as coherent vector:

$$
\boldsymbol{\Gamma}=\operatorname{vec}(\mathbf{C})=\langle\mathbf{E} \otimes \overline{\mathbf{E}}\rangle=\left[\left\langle E_{x} \bar{E}_{x}\right\rangle\left\langle E_{x} \bar{E}_{y}\right\rangle\left\langle E_{y} \bar{E}_{x}\right\rangle\left\langle E_{y} \bar{E}_{y}\right\rangle\right]^{T}
$$

or as Stokes vector:

$$
\boldsymbol{\Sigma}=\gamma \cdot \boldsymbol{\Gamma}=[S Q S U S V S]^{T}
$$

where: $\gamma=\left(\begin{array}{cccc}1 & 0 & 0 & -1 \\ 0 & 1 & 1 & 0 \\ 0 & -i & i & 0 \\ 1 & 0 & 0 & 1\end{array}\right)$ and $\{S Q, S U, S V, S\}$ is the set of unnormalized Stokes parameters involving power flux $S$, linear $\{S Q, S U\}$ and circular $S V$ polarizations of the received wave.

In the following, we will use the Stokes vector formalism, which has the advantage to involve only real quantities. Note that the wave polarization depends on the choice of the reference frame, by a rotation in the view plane. 
By analogy, one can define the measured or "apparent" polarization, as the (time) average of antenna voltage co-variances. Introducing $\mathbf{V}=\left[V_{1} V_{2}\right]^{T}$, where $V_{1}$ and $V_{2}$ are the instantaneous voltages measured by a pair of antennas, one can define:

apparent coherency matrix: $\mathbf{C}_{\mathbf{a}}=\left\langle\mathbf{V} \mathbf{V}^{\mathbf{H}}\right\rangle=\left(\begin{array}{ll}\left\langle V_{1} \bar{V}_{1}\right\rangle & \left\langle V_{1} \bar{V}_{2}\right\rangle \\ \left\langle V_{2} \bar{V}_{1}\right\rangle & \left\langle V_{2} \bar{V}_{2}\right\rangle\end{array}\right)$

apparent coherency vector: $\quad \boldsymbol{\Gamma}_{\mathbf{a}}=\operatorname{vec}\left(\mathbf{C}_{\mathbf{a}}\right)=\langle\mathbf{V} \otimes \overline{\mathbf{V}}\rangle=\left[\left\langle V_{1} \bar{V}_{1}\right\rangle\left\langle V_{1} \bar{V}_{2}\right\rangle\left\langle V_{2} \bar{V}_{1}\right\rangle\left\langle V_{2} \bar{V}_{2}\right\rangle\right]^{T}$

apparent Stokes vector: $\quad \boldsymbol{\Sigma}_{\mathbf{a}}=\gamma \cdot \boldsymbol{\Gamma}_{\mathbf{a}}=\left[S_{a} Q_{a} S_{a} U_{a} S_{a} V_{a} S_{a}\right]^{T}$

Here $S_{a} Q_{a}, S_{a} U_{a}, S_{a} V_{a}, S_{a}$ are the unnormalised, Stokes parameters which fully describe the polarization response of the receiving system. The goal of the model is to determine how $\boldsymbol{\Sigma}_{\mathbf{a}}$ relies on $\boldsymbol{\Sigma}$.

\subsection{Antenna Theory}

Antennas in radio astronomy and telecommunications are most often described by power related quantities as gain directivity, radiation intensity, beam pattern, etc... They also can be described by (far) field related quantities, all of them being expressed in terms of the antenna effective height introduced by Sinclair [1950]. In modern notation, e.g. Macher [2008], it corresponds to the Fourier transform of the current distribution and reads as:

$$
\mathbf{h}(\mathbf{k})=\frac{1}{I_{0}} \int \mathbf{j}(\mathbf{r}) e^{-i \mathbf{k r}} d \mathbf{r}
$$

where: $\mathbf{j}(\mathbf{r})$ is the current density on the antenna, $\mathbf{k}$ is the wave vector and $I_{0}$ the driving current. In the quasi-static frequency range (where the antenna can be assumed to act as a short dipole of length $L=2\|\mathbf{h}\| \ll \lambda$ ), the effective height $\mathbf{h}$ is a constant, real vector. But, in general, $\mathbf{h}$ is complex and depends on both wave frequency and direction [Macher, 2008]. For antennas erected on a spacecraft like Cassini, one has to consider the overall current distribution, including the conductive body of the spacecraft.

The antenna output voltage $\mathrm{V}$ is then linked to the incident wave electric field $\mathrm{E}$, by a scalar product involving the antenna effective height:

$$
V=(P \cdot T \cdot \mathbf{h})^{T} \cdot \mathbf{E}
$$

In Equation (1), let $\mathcal{R}$ be the reference frame in which wave direction and polarisation are finally expressed. $\mathbf{h}$ is the (complex) effective height vector defining the electric properties of the antenna in some reference frame $\mathcal{A}$. 
The $T$ matrix transforms any vector from the antenna reference frame $\mathcal{A}$ to the working reference frame $\mathcal{R}$. Frames $\mathcal{A}$ and $\mathcal{R}$ may be chosen to be different, in particular to handle the case of a spinning spacecraft. Otherwise $T$ is the identity matrix.

The electric field components are expressed in the wave reference frame $\mathcal{W}$, defined such that the $\mathrm{z}$-axis lies along the wave direction and the $\mathrm{x}$-axis is contained in a vertical (meridian) plane of the working reference frame $\mathcal{R}$. The $P$ matrix is the $3 \times 2$ matrix of the projection from the working reference frame $\mathcal{R}$ into the wave reference frame $\mathcal{W}$ : it can be developed into the product of the transformation matrix $P_{E}$ from $\mathcal{R}$ into $\mathcal{W}$ by the projection operator $\mathbb{p}=\left(\begin{array}{ll}1 & 0 \\ 0 & 1 \\ 0 & 0\end{array}\right)$, as: $P=\mathbb{p} \cdot P_{E}$.

\subsection{Introducing the Mueller Matrix}

If one deals with two antennas, the vector $\mathbf{h}$ can be replaced by the matrix $\mathbf{H}$ containing the antenna effective height vectors stacked in columns:

$$
\mathbf{H}=\left[h_{1} h_{2}\right]
$$

and $\mathbf{V}$ is a column vector of voltages:

$$
\mathbf{V}=\left[V_{1} V_{2}\right]^{T}=(P \cdot T \cdot \mathbf{H})^{T} \cdot \mathbf{E}
$$

Following a polarisation formalism used in optics, one can introduce the Jones matrix $\mathcal{J}=(P \cdot T \cdot \mathbf{H})^{T}$ and Equation (2) simply turns out to be:

$$
\mathbf{V}=\mathcal{J} \cdot \mathbf{E}
$$

Finally by using the property $A B \otimes C D=(A \otimes C)(B \otimes D)$ of a matrix outer (Kronecker) product, one finds that true and apparent coherencies are linked by:

$$
\Gamma_{\mathbf{a}}=\langle\mathbf{V} \otimes \overline{\mathbf{V}}\rangle=\mathcal{J} \otimes \overline{\mathcal{J}} \cdot\langle\mathbf{E} \otimes \overline{\mathbf{E}}\rangle=\mathcal{J} \otimes \overline{\mathcal{J}} \cdot \mathbf{\Gamma}=M_{\Gamma} \cdot \mathbf{\Gamma}
$$

in which we introduce the Mueller matrix $M_{\Gamma}=\mathcal{J} \otimes \overline{\mathcal{J}}$.

In the same way, by setting $M_{\Sigma}=\gamma \cdot M_{\Gamma} \cdot \gamma^{-1}$, one finds the relation between true and apparent Stokes vectors:

$$
\Sigma_{a}=M_{\Sigma} \cdot \Sigma
$$

Explicitly, taking into account Equation (2) yields:

$$
\mathbf{M}_{\Sigma}=\gamma \cdot(H \otimes \bar{H})^{T} \cdot(T \otimes T)^{T} \cdot(P \otimes P)^{T} \cdot \gamma^{-1}
$$


The Equation (4) (or Equation (5)) is the fundamental equation of the DF\&Pol problem. It shows that apparent and true polarizations are linked through a linear relationship. The $4 \times 4$ real matrix $\mathbf{M}_{\Sigma}$ is called the "Mueller matrix" of the antenna system.

\subsection{Some Properties of the Mueller Matrix of a Pair of Short Dipoles}

It can be shown [Hovenier, 1994] that the 16 real elements of any Mueller matrix are defined from 7 real independent quantities at most. The interrelations between elements can be revealed by writing that total polarisation must be conserved. Conservation of the total polarization is expressed by setting the quadratic form $\Sigma^{\mathrm{T}} \cdot \mathrm{G} \cdot \Sigma$ to zero, i.e.:

$$
\Sigma_{\mathrm{A}}^{\mathrm{T}} \cdot \mathrm{G} \cdot \Sigma_{\mathrm{A}}=\Sigma^{\mathrm{T}} \cdot \mathrm{M}_{\Sigma}^{\mathrm{T}} \mathrm{GM}_{\Sigma} \cdot \Sigma=0, \text { where } \mathrm{G}=\left(\begin{array}{cccc}
-1 & 0 & 0 & 0 \\
0 & -1 & 0 & 0 \\
0 & 0 & -1 & 0 \\
0 & 0 & 0 & 1
\end{array}\right)
$$

which implies, for a general Mueller matrix, that the relation $M_{\Sigma}^{\mathrm{T}} \cdot \mathrm{G} \cdot \mathrm{M}_{\Sigma}=\left|\mathrm{M}_{\Sigma}\right|^{1 / 2} \cdot \mathrm{G}$ holds.

In the simplified case of a pair of short dipoles, whose effective lengths are real, previous relation separately holds for the real part $\left(S_{a} Q_{a}, S_{a} U_{a}, S_{a}\right)$ and for the imaginary part $\left(S_{a} V_{a}\right)$ of the coherence vector. Combining the two relations provides the following formula which involves the normalized Stokes parameters but, very usefully, does not involve the wave direction:

$$
\frac{\left|V_{a}\right|}{\sqrt{1-Q_{a}^{2}-U_{a}^{2}}}=\frac{|V|}{\sqrt{1-Q^{2}-U^{2}}}
$$

The Equation (6) gives a way to infer on true polarisation of the source without solving the direction finding part of the $\mathrm{DF} \& \mathrm{Pol}$ problem. In particular, it is insensitive to the degenerated case (see below in section 4.5) when the source direction lies in the antenna plane. Additionally, Equation (6) shows that $\left|V_{a}\right| /\left(\sqrt{1-Q_{a}^{2}-U_{a}^{2}}=1\right.$ is always verified for totally polarized waves, and provides a direct measurement of the amount of circular polarisation for a purely circularly polarized wave. Note, as already said, that Equation (6) only holds for the case of real effective lengths, i.e. of short dipoles.

Furthermore, Mueller matrices for short dipoles are defined by only five independent quantities, namely the directions $\left(u_{1}=h_{1} /\left\|h_{1}\right\|, u_{2}=h_{2} /\left\|h_{2}\right\|\right)$ and length ratio of the two antennas $\left(r=\left\|h_{1}\right\| /\left\|h_{2}\right\|\right)$. Since the matrix $H=\left[h_{1} h_{2}\right]$ in Equation $(2)$ is a $3 \times 2$ matrix of rank 2 , for any choice of the antenna frame $\mathcal{A}$, having its z-axis perpendicular to the antenna plane (i.e. having the antenna plane as $\mathrm{x}-\mathrm{y}$ plane), the last row of the $H$ matrix can be set identically equal to zero. For instance, singular value decomposition of $H$ yields:

$$
H=\left(a u_{1}+b u_{2} b u_{1}+a u_{2} \frac{u_{1} \times u_{2}}{\sin \omega}\right) \cdot\left(\begin{array}{ll}
1 & 0 \\
0 & 1 \\
0 & 0
\end{array}\right) \cdot\left(\begin{array}{cc}
r a \sin \omega & -b \sin \omega \\
-r b \sin \omega & a \sin \omega
\end{array}\right)=R_{H} \cdot \mathbb{p} \cdot H_{0}
$$


with ( $\omega$ being the angle between $h_{1}$ and $h_{2}$ )

$$
a=\frac{\sin \left(\frac{\pi}{4}+\frac{\omega}{2}\right)}{\sin \omega} \quad b=\frac{\sin \left(\frac{\pi}{4}-\frac{\omega}{2}\right)}{\sin \omega}
$$

The column vectors of $R_{H}$ form an ortho-normal basis of $\mathcal{A}$. The two first vectors, - along $\mathrm{x}$ - and $\mathrm{y}$-axis of the chosen frame $\mathcal{A}$-, have same bisectors as the antennas $h_{1}$ and $h_{2}$. In the case of perpendicular antennas of the same length, they would simply coincide: $R_{H}=\left(u_{1} u_{2} u_{1} \times u_{2}\right)$ and $H_{0}=I$.

Then Equation (2) can be rewritten as:

$$
\mathbf{V}=H_{0}^{T} \cdot \mathbb{p}^{\mathrm{T}} W^{T} \mathbb{p}^{T} \cdot \mathbf{E}
$$

where $W=P_{E} \cdot T \cdot R_{H}$ is a $3 \times 3$ orthogonal matrix (the one of a rotation), in which only the $2 \times 2$ sub-matrix $W_{0}$, obtained by discarding the last column and the last row of $W$, has to be kept. Finally, $\mathbf{V}=H_{0}^{T} W_{0}^{\mathrm{T}} \mathbf{E}$ and the fundamental Equation (4) becomes:

$$
\begin{aligned}
\boldsymbol{\Sigma}_{a} & =M_{H} \cdot M_{W} \cdot \Sigma \\
M_{H} & =\gamma \cdot\left(H_{0}^{T} \odot H_{0}^{T}\right) \cdot \gamma^{-1} \\
M_{W} & =\gamma \cdot\left(W_{0}^{T} \otimes W_{0}^{T}\right) \cdot \gamma^{-1}
\end{aligned}
$$

in which $M_{H}$ and $M_{W}$ are two $4 \times 4$ square matrices.

If the antennas are already calibrated, that is to say if the opening angle and length ratio of the two antennas have been previously determined, the matrix $M_{H}$ is known and can be inverted. After multiplication of both sides of Equation (7) by $M_{H}^{-1}$, one can correct the apparent Stokes vector as:

$$
\widetilde{\Sigma}_{\mathbf{a}}=M_{H}^{-1} \cdot \Sigma_{a}=M_{W} \cdot \Sigma
$$

Here $\widetilde{\boldsymbol{\Sigma}}_{\mathbf{a}}$ is the "ideal" apparent Stokes vector which would have been measured by a pair of short dipole antennas, orthogonal and of equal lengths. This "ortho-normalisation" of the antenna responses can, once for all, be applied to data.

\subsection{Direction Finding Operations with a Pair of Short Dipoles}

By using the above equations, the various direction finding problems can now be formulated. Let $(\theta, \varphi)$ be the co-latitude and the azimuth of the source in the working frame.

In flight antenna(s) calibration is the process of estimating antennas parameters (i.e. $u_{1}, u_{2}$ and $r$ ) from measurements $\Sigma_{a}$ of a source of known direction $(\theta, \varphi)$ and polarisation $\boldsymbol{\Sigma}$. A minimization procedure for solving Equation (7) can be worked out by 
using a proper set of measurements $\boldsymbol{\Sigma}_{a}$, in which the direction of the calibration source has been varied (see, for example Vogl et al. [2004]).

After the antennas have been fully calibrated, retrieval of parameters of a polarized point source can be attempted. There are six unknowns in Equation (8), namely $S, Q, U, V$ and $\theta, \varphi$. The system of equations, being rank-deficient, cannot be solved in the general case.

Two particular cases are of interest:

Polarized radiation from a known direction: the problem is easily formulated by directly inverting Equation (10), i.e.:

$$
\Sigma=M_{W}^{-1} \cdot \widetilde{\mathbf{\Sigma}}_{a}
$$

However one has to be careful with the last operation, because $M_{W}^{-1}$ is heavily illconditioned for source directions in vicinity of the antenna plane, leading to large uncertainties on derived quantities due to measurement errors. The condition number of $M_{W}^{-1}$, which measures the sensitivity of the solution of Equation (11) to errors in the data, varies as the inverse squared sine of the source elevation above the antenna plane. It exceeds 2 below $45^{\circ}$ (hence in about $60 \%$ of available directions) and reaches 10 at $\sim 20^{\circ}$. The inversion is even impossible $\left(M_{W}^{-1}\right.$ then being singular) when the source direction lies within the antenna plane.

Circularly polarized radiation from an unknown direction: if some additional assumptions can be made on the source polarization, for example assuming that there is no linear polarization $(Q=U=0)$, Equation (10) can be solved in closed form for $\theta, \varphi, V$ and $S$ [Lecacheux, 1978; Cecconi et al., 2005]. There are four distinct solutions corresponding to the symmetry with respect to the antenna plane and to the $\pi$ ambiguity in antenna orientations. However the same restriction as above must apply regarding accuracy of the inversion. Moreover, further analysis shows that uncertainties linked to parallel and perpendicular directions to the antenna plane are in general not equal, because of the non zero correlation between $\theta$ and $S$ in Equation (10). For those reasons, previous published results obtained only in 2-antennas mode (e.g. Cecconi et al. [2009], Lamy et al. [2008]), which assume an a priori, overall angular accuracy of about $2^{\circ}$ in retrieved directions, have to be evaluated with caution.

Finally, Equation (4) can be extended to the case of multiple emitting sources or, more generally, to a continuous sky brightness distribution. Let the source brightness distribution $B(\theta, \varphi)=B(\Omega)$ and its total flux $S=\iint_{\Omega} B(\Omega) d \Omega$. The polarization $Q, U, V$ is supposed to be constant over the whole spatial extent $\Omega$ of the source. Equation (1) yields:

$$
V=(P(\Omega) \cdot H)^{T} \cdot \iint_{\Omega} P\left(\Omega^{\prime}\right)^{T} E\left(\Omega^{\prime}\right) d \Omega^{\prime}
$$

leading to the following generalization of Equation (4): 


$$
\begin{aligned}
M_{\Sigma} & =\gamma \cdot(H \otimes \bar{H})^{T} \cdot(P \otimes P)^{T} \cdot \Im_{\Omega} \cdot \gamma^{-1} \\
\Im_{\Omega} & =\frac{\iint_{\Omega}(P \otimes P)^{T} B(\Omega) d \Omega}{\iint_{\Omega} d \Omega}
\end{aligned}
$$

Equation (10) allows several cases of interest to be easily investigated, namely: a single extended source as a disk of uniform brightness (used in studies of solar type III bursts, e.g. Fainberg et al. [1985]), two point sources of opposite circular polarizations (case of both planetary hemispheres radiating simultaneously), extended sky brightness towards the galactic centre and along the equator (case of sky background estimation), etc...

A detailed analysis of these cases is out of the scope of this work. Let us briefly note the few following recipes. If the brightness distribution of the radio source has a central symmetry around a given direction $(\theta, \varphi)$, but its angular size is neglected in solving Equation (7) instead of Equation (13), one gets biased estimates for $\theta, V$ and $S$, while the azimuth $\varphi$ is still well estimated. Broadly speaking, not measuring the source size pushes the estimated source down to the antenna plane. On the other hand, two point sources at angular distance $D$ will give a response similar to that of a single large source of angular diameter about $D$.

\subsection{Solving DF\&pol Problem in Case of "3 Antennas Mode" Measurement}

Let two apparent Stokes vectors $\Sigma_{a}^{\prime}$ and $\Sigma_{a}^{\prime \prime}$ being obtained from two distinct antenna pairs and assumed to represent two simultaneous observations of the same SKR polarisation state. The unknowns are the source direction $\Omega=(\theta, \varphi)$ and the true polarization state $\Sigma$, involving 6 quantities to be determined from the given 8 measurements. The radiation is here supposed to be emitted by a point source, but a similar analysis could be made by introducing a few more source parameters like, for instance, its size.

Since the problem is overdetermined, we can perform a maximum likelihood estimation of the unknown parameters. The MLE method estimate is then obtained by minimizing the $\chi^{2}$ of residuals (for instance by applying a Levenberg-Marquardt or SVD method), i.e. by solving:

$$
(\widehat{\Omega}, \widehat{\Sigma})=\underset{(\Omega, \Sigma)}{\operatorname{argmin}}\left[\sum_{\Sigma_{a} \in\left\{\Sigma_{a}^{\prime}, \Sigma_{a}^{\prime \prime}\right\}}\left(\frac{\Sigma_{a}-\Sigma_{a_{M O D E L}}(\Omega, \Sigma)}{\sigma_{M O D E L}(\Omega, \Sigma)}\right)^{2}\right]
$$

in which $\Sigma_{a_{M O D E L}}^{\prime}(\Omega, \Sigma)$ and $\Sigma_{a_{M O D E L}}^{\prime \prime}(\Omega, \Sigma)$ are the modelled responses of each antenna pair and $\sigma_{M O D E L}(\Omega, \Sigma)$ the corresponding standard deviation. The only remaining difficulty consists in getting an analytical expression for $\sigma_{M O D E L}^{2}$. Indeed natural or simple weighting are not allowed, since Stokes parameters are correlated by definition. Assuming to have obtained such an expression, $\sigma_{M O D E L}^{2}$ can be approximated, as usually, by replacing the value of $\Sigma$ by the corresponding measured quantities $\Sigma_{a}^{\prime}$ or $\Sigma_{a}^{\prime \prime}$, and the value of the source direction $\Omega$ by that of Saturn's centre. 


\subsection{Statistical Data Model}

The difficulty, pointed out in previous section, can be resolved by analyzing the way in which data are built within the RPWS/HFR receiver. In the HFR real-time processing, "auto" and "cross" measurements are built in the following way:

- take $\mathrm{N}$ voltage samples from both antenna channels and apply narrowband filtering

- form the 4-complex products: $\left\{V_{j} \bar{V}_{k}\right\}_{j, k=1,2}$

- average samples over $\mathrm{N}$, then output the results $\left\langle V_{j} \bar{V}_{k}\right\rangle$

$\mathrm{N}$ determines the fluctuation level and is set appropriately (depending on the chosen frequency band, receiver sweeping mode and integration time). For the RPWS/HFR experiment, it may take values between $\sim 10$ and $\sim 1000$.

Now, given that sample data are stacked in $2 \times \mathrm{N}$ matrix $V$, where $\mathrm{N}$ is the number of samples used for building receiver output, the sample estimate $\mathbf{c}$ of its covariance matrix C is defined by :

$$
\mathbf{c}=\frac{1}{N} \sum_{i=0}^{N-1} V V^{H}
$$

The sample data $V$ are considered independent, centred Gaussian random variables, so that $N \mathbf{c}$ has a central complex Wishart distribution of order 2 and degree N [Goodman, 1963]. Hence, the expectation and covariance of $\mathbf{c}$ are given by:

$$
\mathcal{E}(\mathbf{c})=\mathbf{C} \quad \operatorname{cov}(\mathbf{c})=\frac{2}{N} \overline{\mathbf{C}} \otimes \mathbf{C}
$$

Using Stokes parameters, i.e.:

$$
\sigma=(s q, s u, s v, s)^{T}=\gamma \cdot \operatorname{vec}(\mathbf{c}) \text { and } \mathbf{C}=\frac{1}{2} S\left(\begin{array}{cc}
1+Q & U-i V \\
U+i V & 1-Q
\end{array}\right)
$$

yields expressions for the expectation and covariance matrix of sample estimate $\sigma$ of the Stokes vector $\Sigma$ :

$$
\begin{aligned}
& \mathcal{E}(\sigma)=\Sigma \\
& \operatorname{cov}(\sigma)=\frac{\mathbf{S}^{\mathbf{2}}}{\mathbf{N}}\left(\begin{array}{cccc}
\mathbf{1}+\mathbf{Q}^{2}-\mathbf{U}^{2}-\mathbf{V}^{2} & 2 Q U & & \\
2 U Q & \mathbf{1}-\mathbf{Q}^{2}+\mathbf{U}^{2}-\mathbf{V}^{2} & 2 Q V & 2 Q \\
2 V Q & 2 V U & \mathbf{1}-\mathbf{Q}^{2}-\mathbf{U}^{2}+\mathbf{V}^{2} & 2 U \\
2 Q & 2 U & 2 V & \mathbf{1}+\mathbf{Q}^{2}+\mathbf{U}^{2}+\mathbf{V}^{2}
\end{array}\right)
\end{aligned}
$$

The measured parameters $s q, s u, s v$ and $s$ therefore constitute an unbiased estimate of the observed Stokes parameters. Their variances are respectively equal to the diagonal elements of the above covariance matrix (boldfaced in Equation (16)). 
As previously stated, one also finds that all co-variances are inversely proportional to $\mathrm{N}$, the number of internal samples over which output data are averaged. For N large enough, the estimated Stokes vector $\sigma$ can be considered as a 4-variate gaussian vector (central limit theorem), of expectation and (co)variances given by formulas (16). This should ensure proper weighting for solving the whole estimation problem and allows correct further data averaging (over time and/or frequency).

It is worth noting that formulas (16) will no longer be valid if the assumption that voltage measurements $V$ are i.i.d. Gaussian distributed is actually not satisfied. In particular, it might be the case for SKR (and planetary radio emissions in general), which are known to emit coherent bursts of duration much shorter than currently used integration times.

\subsection{Checking with Real Data}

In order to check the usefulness of the above analysis, a large subset of the RPWS/HFR data is selected, which was obtained in orbit around Saturn from 2006 to early 2010. This subset is based on full polarisation capability (3-antennas mode) of the experiment, and at a single frequency of $290 \mathrm{kHz}$. We choose the $290 \mathrm{kHz}$ frequency in order to get SKR radiation with sufficient signal to noise ratio $(290 \mathrm{kHz}$ is close to the maximum of the SKR spectrum), and measured in a clean and sensitive frequency channel of the receiver.

The selected dataset contains a statistically large number of samples in 3-antennas mode (about 960,000), which were all processed as described in previous section: namely, assuming a point source model, the direction of arrival and the four Stokes parameters are estimated from each individual set of 8 measurements, as well as the quality of the fit and corresponding standard deviations. Since the radio receiver was used in several different combinations of bandwidth and integration time, a direct comparison of estimated and predicted accuracies can be achieved.

As an example, Figure 1 displays a time series of one retrieved SKR parameter, namely the angular deviation of the source direction with respect to the centre of Saturn along its polar axis, during a few days of October 2007, when Cassini was in orbit within 34 to $44 \mathrm{R}_{\mathrm{S}}$ from Saturn. The corresponding (one sigma) estimated error bars are also shown. Two different receiver operating modes were used in sequence at this time. In the less resolved (and more averaging) operating mode, corresponding to grey shaded areas in the plot, both integration time and bandwidth were used to be four times larger, leading to statistical fluctuations of the antenna signal being four times lower. In this mode, the estimated source positions exhibit some well defined variations, larger than the statistical error bar extent. The variations seem to be real: in particular SKR activity exchanges between North and South SKR sources (at about 0300 and 2200 UT on day 30, maybe also visible on late day 28). In the alternate mode, statistical scattering dominates, obfuscating the real source variations or shape. The estimated average standard deviations when using the two modes were $1.8^{\circ}$ and $6.3^{\circ}$, respectively (not far from the predicted factor 4 ratio), in well agreement with our theoretical error analysis of Equation (4). This leads to $\sim 1$ and $\sim 4 \mathrm{R}_{\mathrm{S}}$ (one sigma) error bars at the corresponding distance of Cassini to Saturn, which is smaller and larger than the expected SKR source range from Saturn's centre, respectively. 


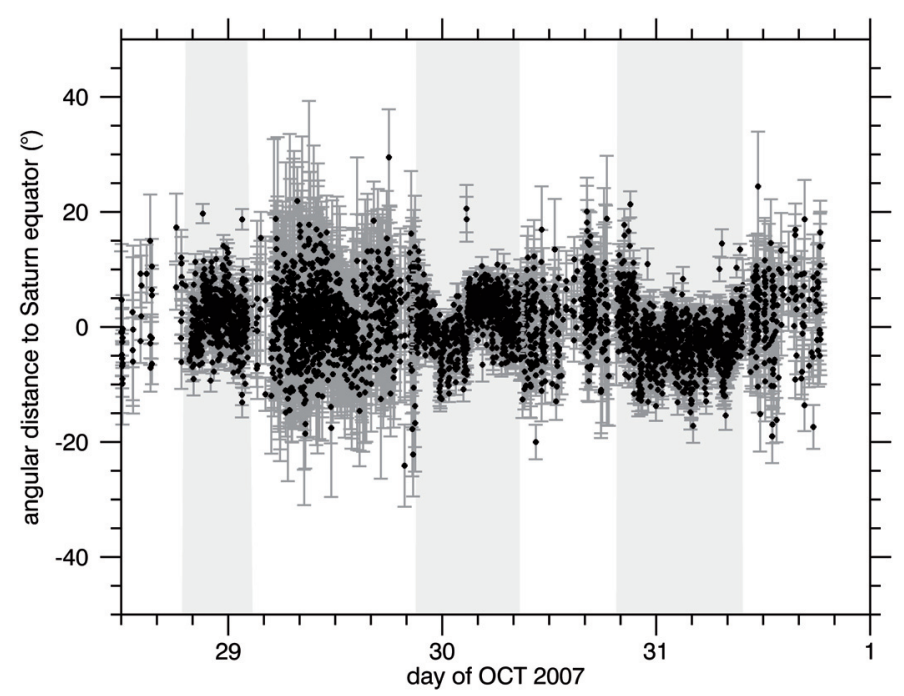

Figure 1: Plot of a time series of estimated angular distances between $290 \mathrm{kHz} S K R$ source regions and Saturn, obtained when two different RPWS acquisition modes were alternately operated, one being faster and using a smaller frequency bandwidth, the other one being slower and using a broader bandwidth. Corresponding time intervals are displayed in white and light grey, respectively. Each dot represents a single sample estimate. Error bars (one standard deviation) are shown in dark grey. Viewed at $\sim 40 \mathrm{R}_{\mathrm{S}}$ from Saturn, SKR radio source morphology can hardly be identified in high resolution mode, due to enlarged statistical fluctuations.

Another more qualitative illustration of the importance of a right choice for the receiver operating mode is given in Figure 2. Each panel displays, in projection onto the sky plane, the cumulative histogram of all measured SKR directions (still, at $290 \mathrm{kHz}$ frequency), when Cassini was at distances from Saturn shorter than $7 R_{S}$. The plot is centred on the direction of Saturn as view from the spacecraft, and Saturn's equator lies along the horizontal axis. The left panel is built from data obtained by using a receiver integration time four times larger (nominally $1 \mathrm{sec}$.) than the integration time used in the right panel (nominally $0.25 \mathrm{sec}$.). While both panels display resembling SKR morphology (a more detailed discussion of it will be given in the next section) and were obtained in similar geometrical and statistical conditions (about 25,000 measurements each), the right one shows significantly more scattering and blur, well in accordance (a factor two in corresponding standard deviations) with theoretical predictions. Note that the representation adopted in Figure 2, of the average distributions of retrieved source directions, does not fully take into account the calculated uncertainties: the faint shades in the background, far from the planet, do not correspond to actually retrieved source regions, but to the contribution of much centered source direction estimates having quite large error bars. 

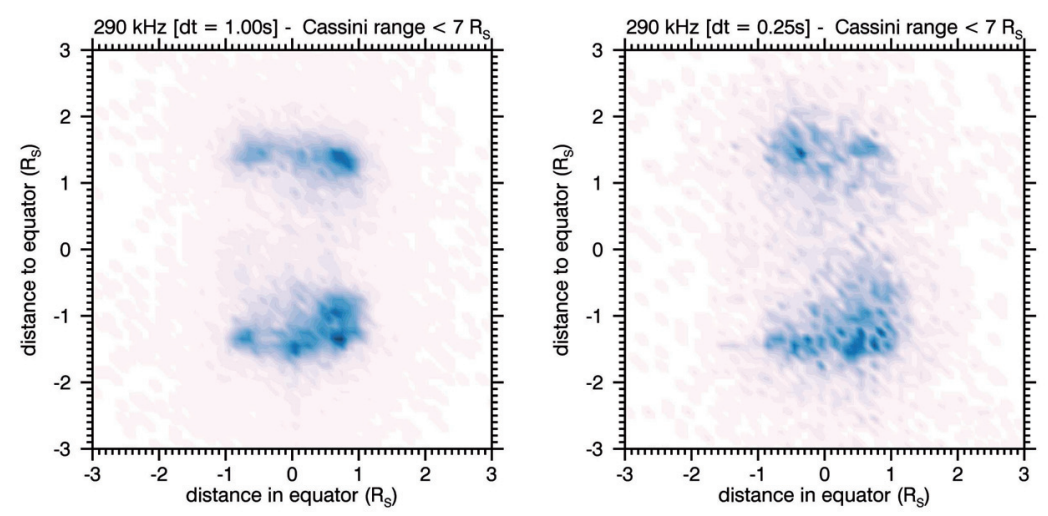

Figure 2: A projection onto the sky view plane of estimated $290 \mathrm{kHz}$ SKR apparent positions, relative to Saturn, when Cassini was at distances closer than $7 \mathrm{R}_{\mathrm{S}}$. Saturn's equator is aligned along horizontal axis, North pole is on the top. The left (resp. right) panel is built from samples integrated over 1 second (resp. 0.25 second).

\section{Some Improved Results on SKR}

At this point, it is appealing to apply the previous calculations and to compare them to our current understanding of SKR source morphology. What would be the appearance of SKR radio source(s) at $290 \mathrm{kHz}$ if observed from a distance of a few planetary radii? As a cyclotron maser, the SKR is radiated near the electron gyro frequency, just above the X-mode cut-off frequency. In regions of negligible plasma density, the two frequencies are approximately equal. The X-mode cutoff at the considered SKR frequency delineates a convex closed surface in space, - the X-surface -, which encloses Saturn and on top of which all possible SKR source locations are lying [Cecconi et al., 2009]. At the radiated frequency of $290 \mathrm{kHz}$, and by modelling Saturn's magnetic field with a dipole having a magnetic moment of 0.21 Gauss. $\mathrm{R}_{\mathrm{S}}^{3}$, the equatorial and polar radii of the $\mathrm{X}$-surface are found to be equal to 1.27 and $1.59 \mathrm{R}_{\mathrm{S}}$, respectively. In addition, if SKR is constrained to occur along some active auroral magnetic field lines (for instance those corresponding to a McIlwain parameter $\mathcal{L}=15$ ), the loci of radio source will reduce to two circles (of radius $\left.0.51 R_{S}\right)$, centred on Saturn polar axis, at equal distance $\left(1.49 R_{S}\right)$ north and south of Saturn's equator.

Furthermore, one can expect that the X-surface will play a major role in subsequent SKR propagation to the observer [Lecacheux, 2005]. Indeed, the X-surface acts as a perfectly reflecting mirror for radio waves. While being an "immaterial" surface (the plasma density is very low there), its optical properties are determined by the coarse and fine structures of Saturn's magnetic field, leading to possible strong refractive and diffractive effects in close vicinity of the SKR source. In particular, if a SKR source comes close to an apparent edge of the X-surface because of the observer's motion with respect to the source, one may expect an "edge focusing" effect (i.e. caustics by reflection) and substantial changes 
in the emission properties at large and small scales ("radio horizon" effect).
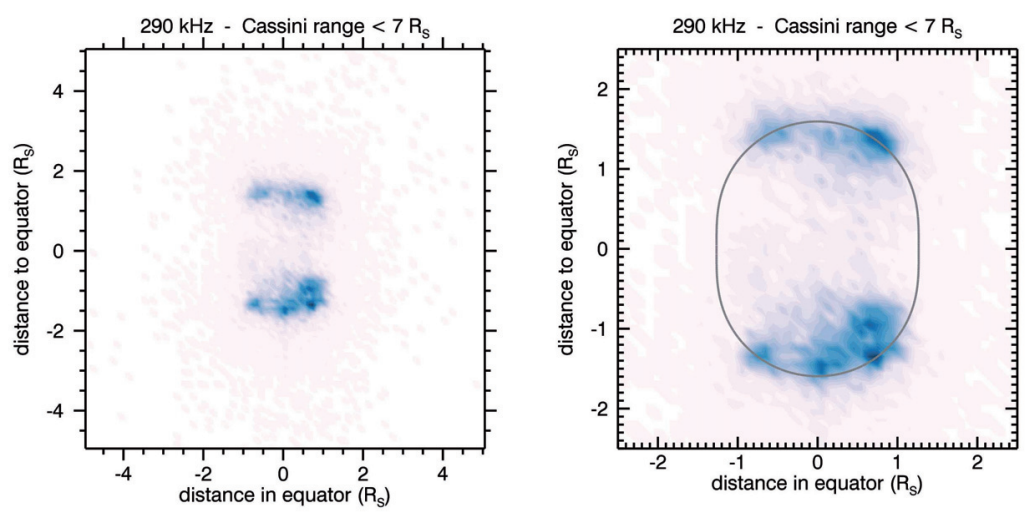

Figure 3: Same as Figure 2, but based on a statistically validated data set containing 5200 estimates. The schematic contour of the $290 \mathrm{kHz} X$-surface is superimposed to observations in the right panel.

In the following, the data set (still at $290 \mathrm{kHz}$ ) is restricted to that obtained when the most accurate receiver operating mode was used and Cassini was at distances closer than $7 \mathrm{R}_{\mathrm{S}}$ from Saturn. The resulting data set consists of 21,000 individual samples spread over 29 different orbits between June 2008 and February 2009. The closest approach from Saturn was $2.5 R_{S}$ and the spacecraft latitude could vary between $-75^{\circ}$ and $+75^{\circ}$. The covered local time range was mainly 2000 to $0000 \mathrm{LT}$, with some excursion to the 0700 to 0900 LT sector.

At this point, it is worth noting that the "single point source model" used for describing the antenna responses, was not applicable all the time. Indeed, when solving Equation 15 , no convergence of the algorithm is obtained for $12 \%$ of the 21,000 considered samples. Regarding the cases of successful convergence, as few as $28 \%$ of the samples (about 5200) fulfill the $\chi^{2}$ condition that residuals are randomly distributed with $95 \%$ confidence level. Very likely, multiple sources or very fast varying radiation are present, which are not well taken into account by our single point source model and then which cause these unsatisfying results.

Taking only into account the 5200 estimates which could be statistically validated allowed us to draw Figure 3. The figure is a mixing of all spacecraft observing geometries with respect to Saturn. In particular the changing spacecraft latitude introduces a perspective effect which is not taken into account. However, the left panel shows, not surprisingly, that SKR sources are confined within two well separated regions, in the north and south of Saturn. They clearly coincide with the expected locations of radio auroral ovals, discussed above. When looking for the circular polarisation (by using Formula 6, for instance), the estimated polarisation sense is always found, without any exception, in accordance with an X-mode emission (LHC at South, RHC at North). This does not confirm, at the 290 $\mathrm{kHz}$ frequency, previous findings of some SKR radiation in O-mode [Lamy et al., 2008]. 


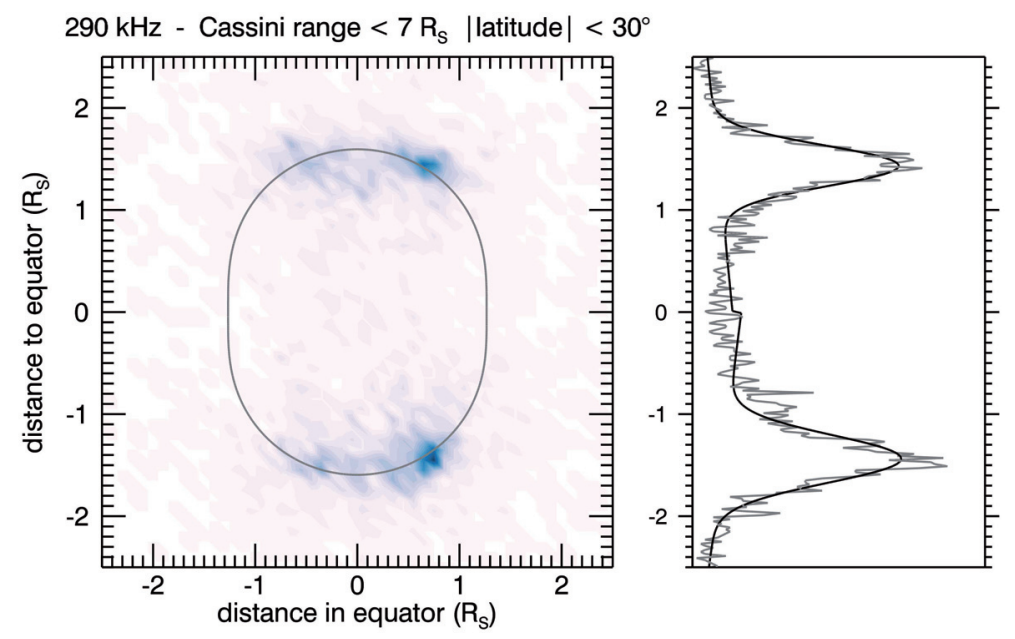

Figure 4: Left: Average distribution, in projection onto the sky view plane, of estimated $290 \mathrm{kHz}$ SKR apparent positions, relative to Saturn, when Cassini was located within $30^{\circ}$ from Saturn's equator. Right: A Gaussian fit (black line) of the histogram of observed SKR source positions along the north-nouth direction provides the estimate $\mathcal{L}=10 \pm 1$ for the McIlwain parameter of SKR active field lines.

The enlarged view in the right panel of Figure 3 compares the observed positions to a schematic contour of the $290 \mathrm{kHz}$ X-surface. There is a quite well agreement. One can note a substantial enhancement of SKR positions at both eastern and western sides of each auroral regions (particularly visible in the northern hemisphere). This is even clearer in Figure 4, where four statistical "spots" can be noticed, occurring at the same distances to the equator, along the east and west limbs of the X-surface.

Restricting the observations to those carried out when Cassini was near Saturn's equator (Figure 4) lowers positional errors due to the perspective effect, and then offers a way to guess at the actual SKR source location. Indeed, by summing up the left panel data over the east-west direction, one can build the distribution of all observed north-south positions (right panel in Figure 4). Then, a bimodal, Gaussian fit (plotted as the black curve in the right panel), provides an estimate of the SKR source mean distance to the equator along Saturn's polar axis, equal to $1.438 \pm 0.015$ (3 sigmas). This leads to the following determination of the McIlwain parameter for SKR active field lines : $\mathcal{L}=10.2 \pm 1.0$. The planetocentric latitude of the corresponding footprint at the cloud level is then $72.4^{\circ} \pm 1.2^{\circ}$, in close agreement with the latitude range found for the UV/IR auroral main ovals [Badman et al., 2006; Grodent et al., 2010].

At this point, the $290 \mathrm{kHz}$ SKR overall radio source can obviously be identified (not surprisingly but, now, with a higher confidence) with the radio auroral ovals defined by mapping the UV/IR auroral ovals along $\mathcal{L} \sim 10$ field lines. The high latitude passes of 

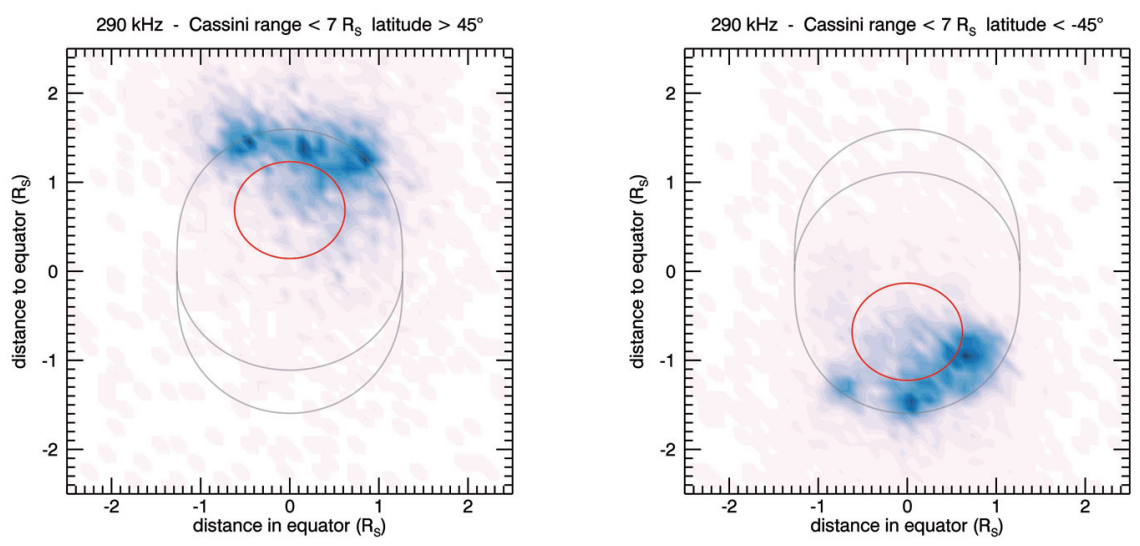

Figure 5: Average distribution, in projection onto the sky view plane, of estimated $290 \mathrm{kHz}$ SKR apparent source positions, relative to Saturn, when Cassini was located at planetocentric latitudes higher than $45^{\circ}$. Schematic contours of the $290 \mathrm{kHz} X$-surface, its equator and $\mathcal{L} \sim 10$ radio ovals are also displayed (for $60^{\circ}$ latitude).

Cassini in 2008-2009 allow the distribution of SKR activity along those ovals to be studied. Figure 5 displays, like Figure 4 for Cassini near Saturn's equator, the distribution of the apparent SKR source positions for Cassini moving above the North (left panel) and the South (right panel) poles, at planetocentric latitudes higher than $45^{\circ}$. In both panels a schematic contour of the $290 \mathrm{kHz}$ X-surface is also displayed (for an observer at $60^{\circ}$ of latitude), as well as the corresponding $\mathcal{L} \sim 10$ radio oval.

By inspection of Figure 5, several striking facts can be noted: i) the apparent SKR activity does occur within a limited range of longitudes, relative to the observer; ii) this activity sector is located on the back side of the planet, beyond Saturn's poles, as viewed by the observer; iii) SKR sources are seen, on average, outside the above defined radio auroral oval; iv) the activity regions, in both hemispheres, look like as being composed of three patches, located at East, at West and on central meridian of the observer, respectively.

In order to perform a more quantitative study, each measurement in Figure 5 was corrected for spacecraft latitude, by simply applying the following geometrical transform which projects observations onto planes parallel to Saturn's equator, assuming some invariant latitude (e.g. corresponding to $\mathcal{L}=10.2$ ) for Saturn's auroral emission,

$$
\begin{aligned}
x^{\prime} & =x \\
y^{\prime} & =\frac{\left(y-y_{0} \cos \left(l a t_{s / c}\right)\right)}{\sin \left(l a t_{s / c}\right)}
\end{aligned}
$$

$\mathrm{x}$ and $\mathrm{y}$ are rectangular coordinates in the view plane, parallel and perpendicular to Saturn's equator, respectively. $\mathrm{x}$ ' and y' are the transformed coordinates, this time as viewed from the polar direction. $y_{0}=1.438$ was previously determined from observations in Saturn's equator and corresponds to $\mathcal{L}=10.2$. 
This procedure yields Figure 6, which finally displays a view of the radio auroral northern and southern ovals as observed from above the corresponding pole. The Cassini spacecraft is located towards the bottom of the figure. One observes that the two distributions of SKR source positions look very similar. Of course, Figure 6 is drawn from Cassini locations far enough from Saturn's equator (30 latitude was chosen as threshold), for which the latter re-projection is well defined (but with uncertainties in $\mathrm{y}$-direction enlarged by a factor $\left.1 / \sin \left(l a t_{s / c}\right)\right)$.
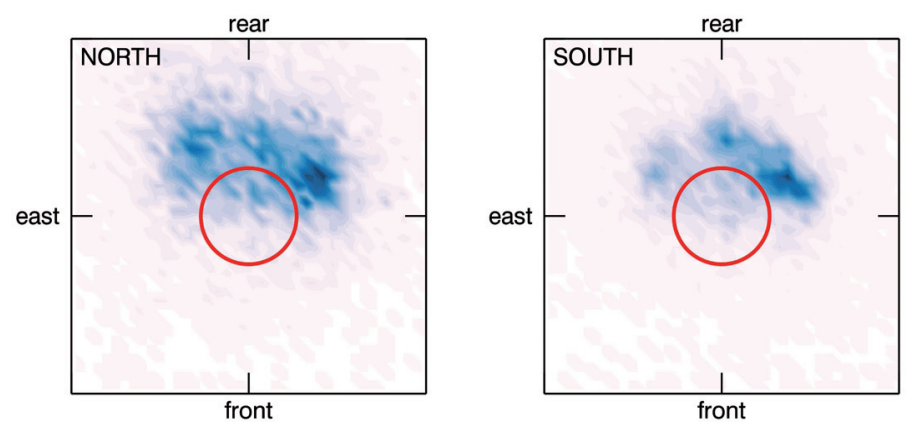

Figure 6: Re-projection of data from Figure 5, taking into account the latitude of the spacecraft. Saturn's polar axis is perpendicular to the page, the observer's direction is downwards. The circle is the $\mathcal{L}=10$ radio auroral oval.

Several factors could change the actual SKR source distribution in longitude: SKR activity may rather depend on (the sun's) local time as inferred by [Lamy et al., 2008], or depend on Saturn's rotation, or be modified by temporal or propagation effects. Since results in Figure 6 are obtained from the analysis of portions of 29 different orbits extending over a nearly one year time interval, Saturn's rotation effects can be ruled out (the histogram in SLS (or SLS3 or whatever) corresponding to observing times being nearly flat over the time range), as well as temporal effects which would average.

Some serious ambiguity remains regarding any dependence on local time, since the Cassini positions along the 29 used orbits are far from being equally distributed in longitude with respect to the Sun. Figure 7 is a redrawing of Figure 6, but in the fixed longitude system relative to the solar direction (1200 LT, at the bottom of each panel). It shows again the existence of a delimited SKR activity sector, but this time on the morning side, within a range of local times which is in agreement with previously published analyses [Kaiser et al., 1984; Lamy et al., 2008].

Then, from presently available data, it is hard to decide from which of Figure 6 or Figure 7 is the right representation of the statistical SKR activity along the auroral ovals.

However, we note that the SKR source distributions in Figure 7 are significantly different in northern and nouthern hemispheres, - with, for instance, a maximum around 0500 LT in North, and 1100 LT in South -, while distributions in Figure 6 look like the same. Now, there is no reason for northern and southern auroral activities to be different with regard 
to hemisphere. They even have been observed to be closely conjugated [Nichols et al., 2010].
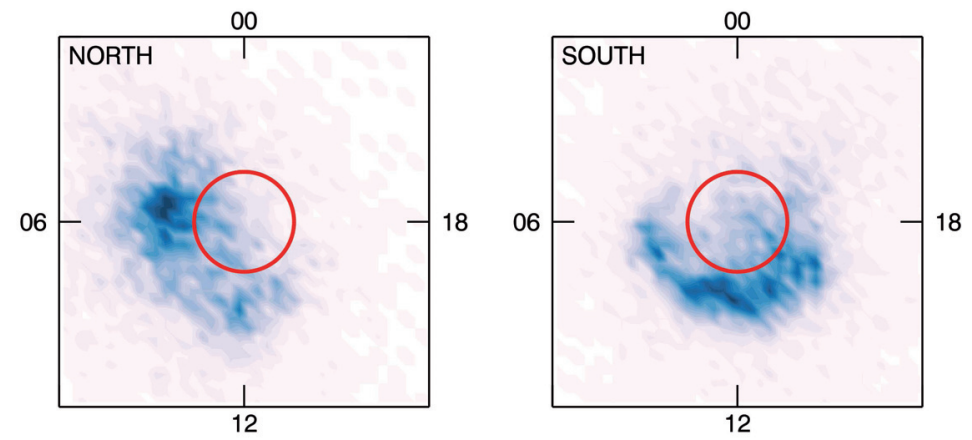

Figure 7: Same as Figure 6, but the origin of the longitude is Sun's direction (1200 LT) at bottom.

Hence we guess that the more compelling distribution is likely the one in Figure 6. Different ranges of spacecraft local times along northern and southern branches of each of the 29 considered orbits, have induced longitude transformations from Figure 6 to Figure 7, leading to an apparent difference between North and South panels in Figure 7.

In other words, it can be concluded that apparent SKR source distributions, for a distant observer, are mainly influenced, in addition to all other known factors (solar angle and morphology of the auroras, planetary spin modulation, etc...), by the position of the observer with respect to the planet. This effect is likely due to a combination of emission beaming and strong propagation effects close to the radio source. Investigation of those effects is out of the scope of the present paper.

Enforcing the last conclusion is the observed shape of the distribution of SKR events showing a high degree of linear polarisation (Figure 8). Fischer et al., 2009] demonstrated that a higher latitude of the observer correlates positively with a higher number of SKR events showing a high degree of linear polarisation, from $0 \%$ in the equator (SKR is purely circularly polarized) up to $80 \%$ at the highest latitudes (SKR is strongly linearly polarized). Figure 8 is a redrawing of Figure 6, with the average degree of SKR linear polarisation plotted in colored scale. One clearly sees that the east and west patches of emissions display pure circular polarisation (like when viewed from the equator), while high linear polarisation is more or less confined to the patch on the central meridian and to the regions well behind the poles. By re-plotting data in function of local time, like in Figure 7 , polarisation events get mixed, without any clear ordering in local time. 


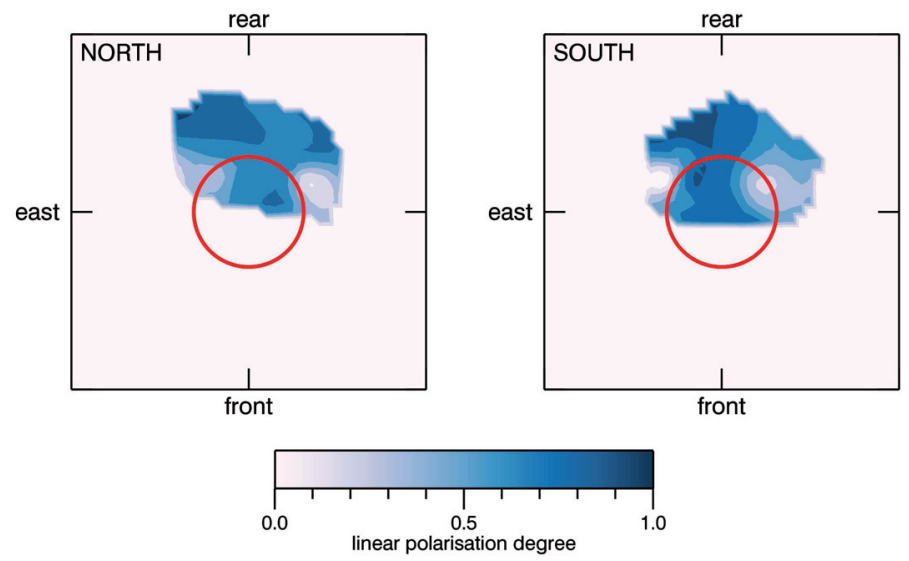

Figure 8: Same as Figure 6, but displaying linear polarization degree in colored scale. Note the two patches of pure circular polarization (linear polarization degree equal to zero) to the east and to the west of the line of sight. The regions with a high degree of linear polarization are concentrated in between and beyond the poles.

\section{Summary}

The capabilities of the RPWS/HFR radio astronomy instrument aboard Cassini spacecraft have been analyzed regarding direction finding and polarisation retrieval of observed radio emissions. The configuration of the experiment is close to the ideal instrumental design which would allow a maximal science return by using simple wire antennas erected on a spacecraft: namely three, non coplanar monopoles and the full set of corresponding two-way correlators [Lecacheux et al., 1979].

In the frame of the coherence theory of electromagnetic waves, a simple, but quite general formalism has been developed which is linking the observed radio source(s) properties to the measured quantities (the output of correlators). Both can be expressed in terms of "true" and "apparent" Stokes vectors, interrelated through a linear relationship, described by special matrices, the Mueller matrices.

The formalism allows most of the direction and polarization retrieval problems to be easily analyzed, implemented in software, then solved. It also permits a full statistical error analysis of the derived quantities. The latter analysis is necessary for assessing the consistency and physical meaning of obtained results. The magnitude and correlation of the uncertainties on derived parameters appear to depend notably on spacecraft orientation as well as on the chosen time-bandwidth product of spectral analyzers. Both have to and can be optimized, according to the favored science objective.

For illustration, a limited but representative analysis of some SKR direction measurements with respect to Saturn's environment is presented. The analysis is limited to only one SKR frequency $(290 \mathrm{kHz})$ and to Saturn ranges closer than $7 \mathrm{R}_{\mathrm{s}}$, in order to maximize the 
spatial resolution of the method. The data spans about one year and uses observations along portions of 29 different Cassini orbits, lying within a large domain of longitudes and, especially, latitudes around Saturn. Our findings were the following:

- A substantial proportion of the data must be rejected, because it is statistically inconsistent with the basic assumption that the observed SKR would come from a single, steady emitting point source.

- The remaining part (several thousands of single estimated directions) constrains SKR sources to the "radio auroral oval", defined as the mapping of Saturn's main $\mathrm{UV} / \mathrm{IR}$ auroral oval along $\left(\mathcal{L} \sim 10\right.$ or invariant latitude $\left.\sim 72^{\circ}\right)$ magnetic field lines. The deduced circular polarisation senses are in agreement with a pure $\mathrm{X}$-mode emission.

- On average, the source positions were found to lie on the radio auroral oval, but at preferred locations, which mostly depend on the observer's direction with respect to Saturn. These locations are on the east and west ends of auroral ovals, when Cassini is in Saturn's equator. They are on the most central, back part, when Cassini is at high latitudes. In addition, side and most central locations on the radio auroral oval closely correspond to observed circularly and linearly polarized SKR emissions, respectively.

The latter finding, which has to be confirmed by further analysis, suggests the existence of significant propagation effects, being able to constrain the visibility of emission (strong refraction) or to change the polarisation (mode coupling).

\section{References}

Badman, S. V., S. W. H. Cowley, J.-C. Gérard, and D. Grodent, A statistical analysis of the location and width of Saturn's southern auroras, Ann. Geophys., 24, 3533-3545, 2006 .

Born, M. and E. Wolf, Principles of optics : electromagnetic theory of propagation, interference and diffraction of light, Cambridge University Press, New York, 1999.

Cecconi, B. and P. Zarka, Direction finding and antenna calibration through analytical inversion of radio measurements performed using a system of two or three electric dipole antennas on a three-axis stabilized spacecraft, Rad. Sci., 40, 3, 2005.

Cecconi, B., L. Lamy, P. Zarka, R. Prangé, W.S. Kurth, and P. Louarn, Goniopolarimetric study of the revolution 29 perikrone using the Cassini Radio and Plasma Wave Science instrument high-frequency radio receiver, J. Geophys. Res., 114, A3, A03215, 2009.

Fainberg, J., S. Hoang and J.-L. Steinberg, Measurements of Distributed Polarized Radio Sources from Spinning Spacecraft - Effect of a Tilted Axial Antenna - ISEE-3 Application and Results, Astron. Astrophys., 153, 2, 145-150, 1985. 
Fischer, G., B. Cecconi, L. Lamy, S.-Y. Ye, U. Taubenschuss, W. Macher, P. Zarka, W.S. Kurth, and D. A. Gurnett, Elliptical polarisation of Saturn Kilometric radiation observed from high latitudes, J. Geophys. Res., 114, A08216, 2009.

Goodman, N.R., Statistical analysis based on a certain multivariate complex Gaussian distribution (An introduction), Ann. Math. Stat., 34, 152-177, 1963.

Grodent, D., A. Radioti, B. Bonford, and J.-C. Gérard, On the origin of Saturn's outer auroral emission, J. Geophys. Res., 115, A08219, 2010.

Gurnett, D. A., et al. (29 co-authors), The Cassini Radio and Plasma Wave Investigation, Space Sci. Rev., 114, 395-463, 2004.

Hamaker, J. P., J. D. Bregman and R. J. Sault, Understanding radio polarimetry, Astron. Astrophys., 117, 137-147, 1996.

Hovenier, J. W., Structure of a general pure Mueller matrix, App. Opt., 33, 8318-8324, 1994.

Kaiser, M. L., M.D. Desch, W.S. Kurth, A. Lecacheux, F. Genova, B. M. Pedersen, and D.R. Evans, Saturn as a radio source, in Saturn, edited by T. Gehrels, and M. S. Matthews, University of Arizona Press, Tucson, 378-415, 1984.

Lamy, L., P. Zarka, B. Cecconi, R. Prangé, W.S. Kurth, and D. A. Gurnett, Saturn kilometric radiation: Average and statistical properties, J. Geophys. Res., 113, A07201, doi:10.1029/2007JA012900, 2008.

Lamy, L., P. Schippers, P. Zarka, B. Cecconi, C. S. Arridge, M. K. Dougherty, P. Louarn, N. André, W. S. Kurth, R. L. Mutel, D. A. Gurnett, and A. J. Coates, Properties of Saturn kilometric radiation measured within its source region, Geophys. Res. Lett., 37, L12104, doi:10.1029/2010GL043415, 2010.

Lamy, L., B. Cecconi, P. Zarka, P. Canu, P. Schippers, W. S. Kurth, R. L. Mutel, D. A. Gurnett, D. Menietti, and P. Louran, Emission and propagation of Saturn kilometric radiation: Magnetoionic modes, beaming pattern, and polarization state, J. Geophys. Res., 116, A04212, 2011.

Lecacheux, A., Direction Finding of a Radiosource of Unknown Polarization with Short Electric Antennas on a Spacecraft, Astron. Astrophys., 70, 701-706, 1978.

Lecacheux, A., Polarization aspects from planetary radio emissions, in Planetary Radio Emissions II, edited by H. O. Rucker, S. J. Bauer, and B. M. Pedersen, Austrian Academy of Sciences Press, Vienna, 311-325, 1988.

Lecacheux, A., The "Radio Horizon" Effect as a Possible Explanation of the Planetary Auroral Radio Emission Phenomenology, in Planetary Radio Emissions VI, edited by H. O. Rucker, W.S. Kurth and G. Mann, Austrian Academy of Sciences Press, Vienna, 151-152, 2005.

Lecacheux, A., C. C. Harvey, and A. Boischot, Source localization and polarization determination in low frequency satellite radio astronomy, Ann. Telecomm., 34, 253-265, 1979 . 
Macher, W., Transfer matrix description of multi-port antennas and its application to the Mars Express/MARSIS radar, University of Graz, Austria, PhD. IWF-182, 2008.

Nichols, J. D., S. W. H. Cowley, and L. Lamy, Dawn-Dusk Oscillation of Saturn's Conjugate Auroral Ovals, Geophys. Res. Lett., 37, L24102, 2010.

Sinclair, G., The transmission and reception of elliptically polarized waves, Proc. IRE, 38, 148-151, 1950.

Thompson, A. R., J. M. Moran, and G. W. Swenson, Interferometry and Synthesis in Radio Astronomy, John Wiley \& Sons, 2007.

Vogl, D. F., B. Cecconi, W. Macher, P. Zarka, H.-P. Ladreiter, P. Fedou, A. Lecacheux, T. Averkamp, G. Fischer, H. O. Rucker, D. A. Gurnett, W. S. Kurth, and G. B. Hospodarsky, In-flight calibration of the Cassini-Radio and Plasma Wave Science (RPWS) antenna system for direction-finding and polarization measurements, J. Geophys. Res., 109, A09S17, 2004.

Warwick, J. W., J. B. Pearce, D. R. Evans, T. D. Carr, J. J. Schauble, J. K. Alexander, M. L. Kaiser, M. D. Desch, B. M. Pedersen, A. Lecacheux, G. Daigne, A. Boischot, and C.H. Barrow, Planetary Radio Astronomy observations from Voyager 1 near Saturn, Science, 212, 239-243, 1981. 
